\title{
Self-Assessments of Standardized Scalp Massages for Androgenic Alopecia: Survey Results
}

Robert S. English Jr. • James M. Barazesh

Received: November 25, 2018 / Published online: January 22, 2019

(C) The Author(s) 2019

\begin{abstract}
Introduction: Standardized scalp massages (SSMs) improve hair thickness in nonbalding men, but their effects on androgenic alopecia (AGA) have not yet been evaluated. The objective of this study was to investigate the effect of SSMs on self-assessed AGA sufferers (SAGASs).

Methods: Between October 2016 and October 2017, 1899 SAGASs searching online for hair loss treatments beyond AGA management drugs accessed literature explaining SSMs as a potential therapy for AGA, then watched a demonstration video detailing twice-daily, 20-min SSMs segmented by three rotational scalp regions using hand-generated presses, pinches, and stretches. In December 2017,
\end{abstract}

Enhanced Digital Features To view enhanced digital features for this article go to https://doi.org/10.6084/ m9.figshare.7577618.

Electronic supplementary material The online version of this article (https://doi.org/10.1007/s13555019-0281-6) contains supplementary material, which is available to authorized users.

R. S. English Jr. ( $ه)$

PerfectHairHealth.com, 180 Steuart St, P.O. \#193606, San Francisco, CA, USA e-mail: rob@perfecthairhealth.com

J. M. Barazesh

Department of Civil and Environmental

Engineering, University of California at Berkeley,

Berkeley, CA, USA
SAGASs were contacted once to participate in a retrospective survey study to assess SSM adherence and hair changes. Age, gender, hair loss region and gradient, diet, supplement and topical use, AGA management drug use, estimations for minutes daily and months of massaging, and self-perceived hair changes were reported. Some participants also submitted photosets documenting hair changes throughout SSM adherence.

Results: A total of 340 (17.9\%) respondents completed the survey, and 327 (17.2\%) reported attempting the SSMs. SSM participants reported a median daily massage effort of 11-20 min and mean adherence of $7.4 \pm 6.6$ months, with $68.9 \%$ reporting hair loss stabilization or regrowth. Estimated minutes daily, months, and total SSM effort (i.e., minutes daily $x$ months) were positively associated with selfperceived hair changes. On average, perceived hair loss stabilization and regrowth occurred after $36.3 \mathrm{~h}$ of SSM effort. Results did not vary across age, gender, Norwood gradient, or concomitant supplement, topical, finasteride, minoxidil, or microneedling use. However, hair change improvements were marginally lower for participants reporting diffuse versus frontal/ temporal or vertex thinning.

Conclusions: While further research is warranted, these results align with previous findings and suggest the potential for SSMs to improve AGA. 
Keywords: Androgenic alopecia; Hair loss; Massage; Mechanotransduction; Soft tissue manipulation

\section{INTRODUCTION}

Androgenic alopecia (AGA) is a common hair disorder that presents as patterned, progressive hair loss across the frontotemporal and vertex scalp regions of men and women. AGA advances with age and diminishes body image satisfaction among sufferers $[1,2]$. Its etiology is purported to be polygenic and androgenic, but the underlying molecular mechanisms governing its onset and progression are not fully understood [3].

AGA's pathobiological suspects include androgens (i.e., $5 \alpha$-dihydrotestosterone), haircycle-regulating signaling proteins (i.e., interleukin 6 , transforming growth factor beta 1 and 2 ), inflammatory fatty acid derivatives (i.e., prostaglandin D2), signaling pathways and pathway inhibitors (i.e., Wnt/ $\beta$-catenin, dickkopf-1), and concomitant morphology (i.e., vascularity, perifollicular fibrosis) [3, 4]. Two Food and Drug Administration (FDA)-approved AGA management drugs-finasteride and minoxidil-reduce $5 \alpha$-dihydrotestosterone and improve vasodilation in scalp tissues, respectively [3]. Clinical studies suggest a response rate to finasteride of $80-90 \%$, with efficacy generally limited to stopping AGA progression along with a $10 \%$ increase in hair count over 2 years [5, 6]. Photographic assessments of male minoxidil users suggest some hair regrowth in $38.4 \%$ of participants [7], while usage reports note that $95 \%$ voluntarily discontinue treatment by 1 year-with $66.5 \%$ stopping due to "low effect" [8]. Consequently, demand remains high for novel and effective AGA therapies.

In the last decade, AGA treatment trials have expanded to stimulation-based therapies (SBTs) including platelet-rich plasma therapy [9], microneedling [10], and polydioxanone monofilament threading [11]. SBTs activate wound-healing responses and improve AGA outcomes purportedly by releasing plateletderived growth factor and vascular endothelial growth factor (VEGF), as well as activating anagen-initiating $\mathrm{Wnt} / \beta$-catenin and dermal papilla (DP) stem cells [9-12]. Soft tissue manipulation (i.e., massaging) may share therapeutic overlap with SBTs. When exposed to mechanical force, cells respond in a dose-dependent manner by altering gene expression to initiate cellular damage or repair [13]. Mechanotherapy is the manipulation of cellular responses by way of stretching, contraction, or compression for therapeutic effect [14]. Depending on the device, duration, and technique, soft tissue manipulation may activate wound-healing and mechanotherapeutic mechanisms to elicit positive outcomes in a variety of disorders.

In animal models, massage therapy increases VEGF-A and neocollagenesis in exercised tendons [15], improves angiogenesis and attenuates fibrosis onset postinjury [16], and is hypothesized to enhance nutrient delivery to fibroblasts and help maintain tissue integrity after stress exposure [17]. In humans, cyclical tissue stretch- and compression-based therapies decrease scar thickness and improve skin elasticity following soft tissue injury [18]. Interestingly, standardized scalp massages (SSMs) increase hair thickness in nonbalding men [14]. Human scalp DP cell stretching in vitro upregulates anagen-associated noggin, SMAD4, interleukin 6 signal transducer, and bone morphogenetic protein 4 while downregulating catagen-associated interleukin 6 [14]. However, the effects of SSMs on AGA have not yet been investigated.

This survey study evaluated the relationship between SSMs and self-perceived hair changes in self-assessed AGA sufferers (SAGASs). Between October 2016 and October 2017, 1899 SAGASs searching online for AGA therapies outside of FDA-approved drugs navigated to PerfectHairHealth.com and accessed materials detailing SSMs as a potential AGA therapy along with instructions for their implementation. In December 2017, material-accessing SAGASs were contacted and asked to participate in a questionnaire to assess SSM adherence. We sought to determine if adherence to twice-daily, 20-min, hand-generated SSMs was associated with self-perceived hair changes. We also examined if the minutes daily by which 
participants reached total SSM efforts influenced results. Secondary objectives included identifying relationships between SSMs and self-perceived hair changes across reported ages, genders, diets, hair loss regions and gradients, adjunct SBTs (i.e., microneedling), and AGArelated supplement, topical, and drug (i.e., minoxidil and finasteride) use.

\section{METHODS}

\section{Participants}

Between May 2014 and September 2015, SAGASs searching online for AGA therapies beyond FDA-approved AGA management drugs navigated to PerfectHairHealth.com and purchased literature and a demonstration video detailing massages as a potential AGA therapy. The materials encouraged two 20-min scalp massages daily, a commitment to massaging for at least 10 months, and a recommendation to record hair changes by taking monthly photographs at comparable lightings, angles, and distances with similar hair styles, lengths, and wetness. SAGASs were offered email support for massage-related questions and to foster adherence. From 2014 to 2016, some massage-adhering SAGASs submitted photographs showing positive hair changes, then participated in qualitative email and video interviews to describe their techniques and adherence. In October 2016, the literature and demonstration video were updated to standardize the massages based on the techniques of therapy-responding SAGASs (Materials S1, Materials S2).

In December 2017, 1899 SAGASs who had purchased the updated literature and demonstration video between October 2016 and October 2017 were contacted once through email to participate in an online survey. Entries were limited to one response per computer to prevent repeat submissions. One hundred eighteen $(6.2 \%)$ contactees had also viewed the original literature and demonstration videopotentiating the evaluation of long-term (>1 year) massage adherence. For a flowchart detailing participant collection, see the supplementary material (Fig. S1).

Participants reported age, gender, region of hair loss (i.e., frontal/temporal, vertex, diffuse), AGA gradient, and confirmed if they had read the literature, watched the demonstration video, and attempted the SSMs. SSM participants then estimated their minutes daily and months of SSM adherence, as well as their diet and concomitant use of AGA-related topicals, supplements, drugs, and SBTs (Materials S3). Self-perceived hair changes were reported using a five-point scale (i.e., -2 to $+2 ; 0=$ hair loss stabilization)-amended from a seven-point self-assessment scale used in AGA microneedling studies [10].

\section{SSMs}

The SSMs specified twice-daily, 20-min, handgenerated scalp massages spaced $12 \mathrm{~h}$ apart. SSM sessions were segmented into one of three rotational scalp regions (frontal/temporal, vertex, sides/back) and divided between hand presses, stretches, and pinches. SSM sessions were as follows: $3 \mathrm{~min}$ of warm-up (cyclical presses at $5-10 \mathrm{lb}$ of downward force per hand across the entire scalp), followed by $17 \mathrm{~min}$ of region-specific tissue manipulation:

- 6 min of scalp skin pinches: single-handed or double-handed pinches with grip spaced 1-2 inches apart, applying 5-10 lb of downward force per hand, and holding 1-2s before moving onto adjacent scalp skin 1-2 inches from previous pinch;

- 6 min of scalp skin presses: knuckle- or palmbased skin presses applying 10-20 lb of downward force, firmly planting against and manipulating scalp skin for 1-2 s before moving onto adjacent scalp skin 1-2 inches from the previous press;

- 5 min of scalp skin stretches: single-handed or double-handed skin stretches with grip spaced 1-2 inches apart, applying firm but comfortable pressure and holding for 1-2 s before moving onto adjacent scalp skin 1-2 inches from previous stretch.

The updated literature and demonstration video recommended a commitment to SSMs of 
at least 10 months. The materials also emphasized the importance of acute inflammation generation (as evidenced by mild erythema by the end of an SSM session) and tissue stretching-to mimic potential mechanistic overlap from SBTs and mechanotherapies previously shown to have therapeutic effects on human scalp hair (Materials S1, Materials S2).

\section{Bias}

Since SSM participation and hair changes were self-assessed, we sought to quantify potential bias in reporting. Analyses for bias exploration can be found in the supplementary material (Materials S4).

\section{Data}

Prior to analyses, email addresses and openended commentary were removed to prevent the sharing of personally identifiable information. All nonnumeric variables were recoded into numeric values. Analyses were conducted in SPSS v24 using linear multiple regression techniques. All procedures performed in studies involving human participants were in accordance with IntegReview IRB, who granted an exemption from Institutional Review Board oversight in accordance with 45 CFR 46.101(b), and with the 1964 Helsinki Declaration and its later amendments or comparable ethical standards. Informed consent was obtained from all individual participants included in the study.

\section{RESULTS}

\section{Preliminary Analyses}

Of the 1899 survey contactees, 360 (19.0\%) responded, 340 (17.9\%) completed the survey, and $327(17.2 \%)$ reported attempting at least one SSM. Of the 327 SSM participants, 296 (90.5\%) identified as male, 29 (8.9\%) identified as female, and 106 (32.4\%) reported SSM adherence $\geq 10$ months. In total, 319 (97.6\%) SSM participants reported all estimations for
SSM adherence, self-perceived hair changes, and at least one hair loss type/region. For purposes of consistency across analyses, figures (unless otherwise noted) present data of these 319 participants.

Daily SSM efforts spanned from $0-10$ to $41+$ min. Median daily massage time was 11-20 min, with a frequency distribution of 0-10 (25.8\%), 11-20 (23.8\%), 21-30 (17.6\%), $31-40(22.9 \%)$, and $41+(10.3 \%)$ min daily. SSM participation ranged from 0 to $37+$ months with an average adherence of $7.4 \pm 6.6$ months. Total SSM effort (minutes daily $\times$ months) ranged from 0 to $647.5 \mathrm{~h}$, with median and mean effort of 50.0 and $85.6 \mathrm{~h}$, respectively. SSM participants reporting $0-10 \mathrm{~min}$ daily averaged a total SSM effort of $14.3 \mathrm{~h}$, whereas those reporting $41+\min$ daily averaged $182.7 \mathrm{~h}$-demonstrating that, on average, greater daily massage time resulted in greater total SSM effort (Fig. 1).

Of the 319 SSM participants, 119 (37.3\%) reported hair loss stabilization (0) and 101 $(31.7 \%)$ reported slight or significant hair regrowth $(+1$ or +2$)$. SSM participants reported a mean self-perceived hair change of 0.03 , indicating that independent of total SSM effort, the average participant perceived hair loss stabilization (Fig. S4).

\section{Effects of SSM Participation on Self- Perceived Hair Changes}

Increasing total SSM effort was associated with increased self-perceived hair change (Fig. 2). On average, self-perceived hair changes beyond stabilization were achieved after $36.3 \mathrm{~h}( \pm 13 \mathrm{~h}$, $85 \%$ confidence interval) of SSM effort. Interpretation of the trendline beyond $200 \mathrm{~h}$ requires caution since the data distribution is positively skewed, with SSM efforts in the bottom $10 \%$ spanning $0-5 \mathrm{~h}$ and the top $10 \%$ spanning 210-647 h. Accordingly, a fairer representation of the data is achieved by binning total SSM efforts by $10 \%$ increments, with bin size selected to have equal numbers of participants $(n=\sim 32)$. The top $20 \%$ of total SSM effort participants $(\geq 152 \mathrm{~h}$ ) reported self-perceived hair changes of 0.36 and the bottom $20 \%$ 


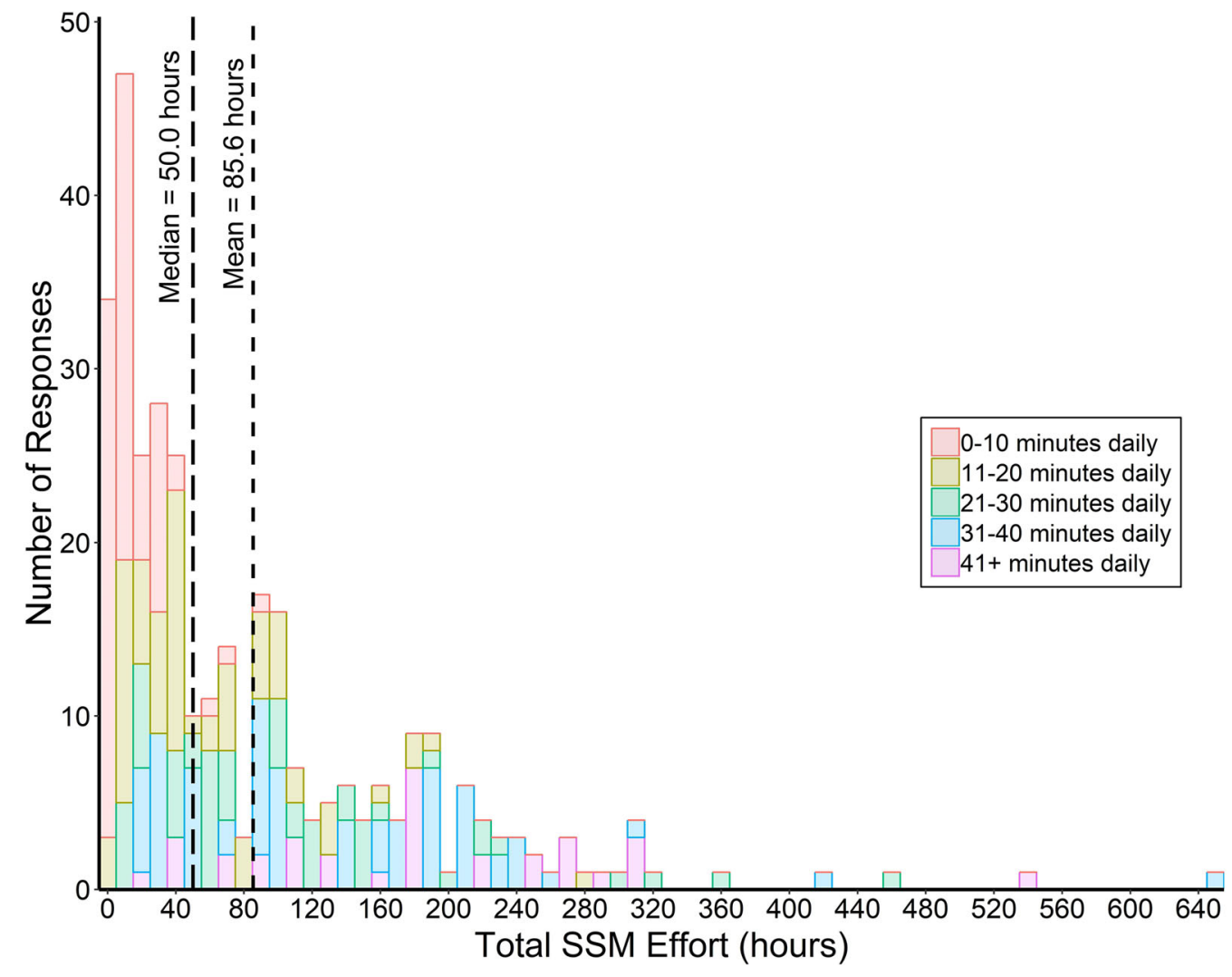

Fig. 1 Total SSM effort (estimated months $\times$ minutes daily) organized by SSM participant response frequencies. Color codes indicate estimated minutes daily of massage time by which total SSM effort was achieved

$(<12.5 \mathrm{~h})$ reported -0.52 , with positive results achieved and maintained at total SSM efforts in the top 50\% ( $\geq 50 \mathrm{~h}$ ) (Fig. S5).

\section{Effects of Minutes Daily on Self-Perceived Hair Changes throughout Total SSM Effort}

We sought to determine if the minutes daily by which participants reached a total SSM effort influenced self-perceived hair changes. Participants were binned into total SSM effort groups of low (25-45 h), medium (85-120 h), and high (180-250 h), then segmented by minutes daily to evaluate mean hair changes and SSM efficiencies (i.e., hair change/total SSM hours per participant) (Fig. S4). Low-effort participants reporting $0-10,11-20$, and $21-30 \mathrm{~min}$ daily achieved similar total SSM efforts of 32.5 $(n=13), 38.0(n=22)$, and $30.7 \mathrm{~h}(n=11)$, but hair changes of $-0.21,0.00$, and 0.545 , respectively. From 0-10 to $21-30 \mathrm{~min}$ daily, SSM efficiencies in the low-effort group increased from -0.006 to $0.018 \mathrm{units} / \mathrm{h}$, respectively. Results were consistent for medium- and higheffort groups. From 11-20 to 31-40 min daily, medium-effort participants reported hair changes of $0.20(n=15$; effort $=105 \mathrm{~h})$ and 0.30 $(n=30$; effort $=111 \mathrm{~h})$, respectively. For higheffort participants, increases in hair changes and SSM efficiencies were observed from 30-41 $(n=18$; hair change $=0.11$; efficiency $=0.0005$ units/hour) to $41+\min$ daily $(n=11$; hair change $=0.64 ; \quad$ efficiency $=0.003$ units $/ \mathrm{h}$ ) indicating that, on average, hair changes improved with more minutes daily of massaging across effort groups.

However, since total SSM effort is a function of minutes daily $\times$ months, binning participants by effort creates significant variance in the average months of adherence at minutes daily endpoints. For instance, while the loweffort group completed $25-45 \mathrm{~h}$ of massaging, 


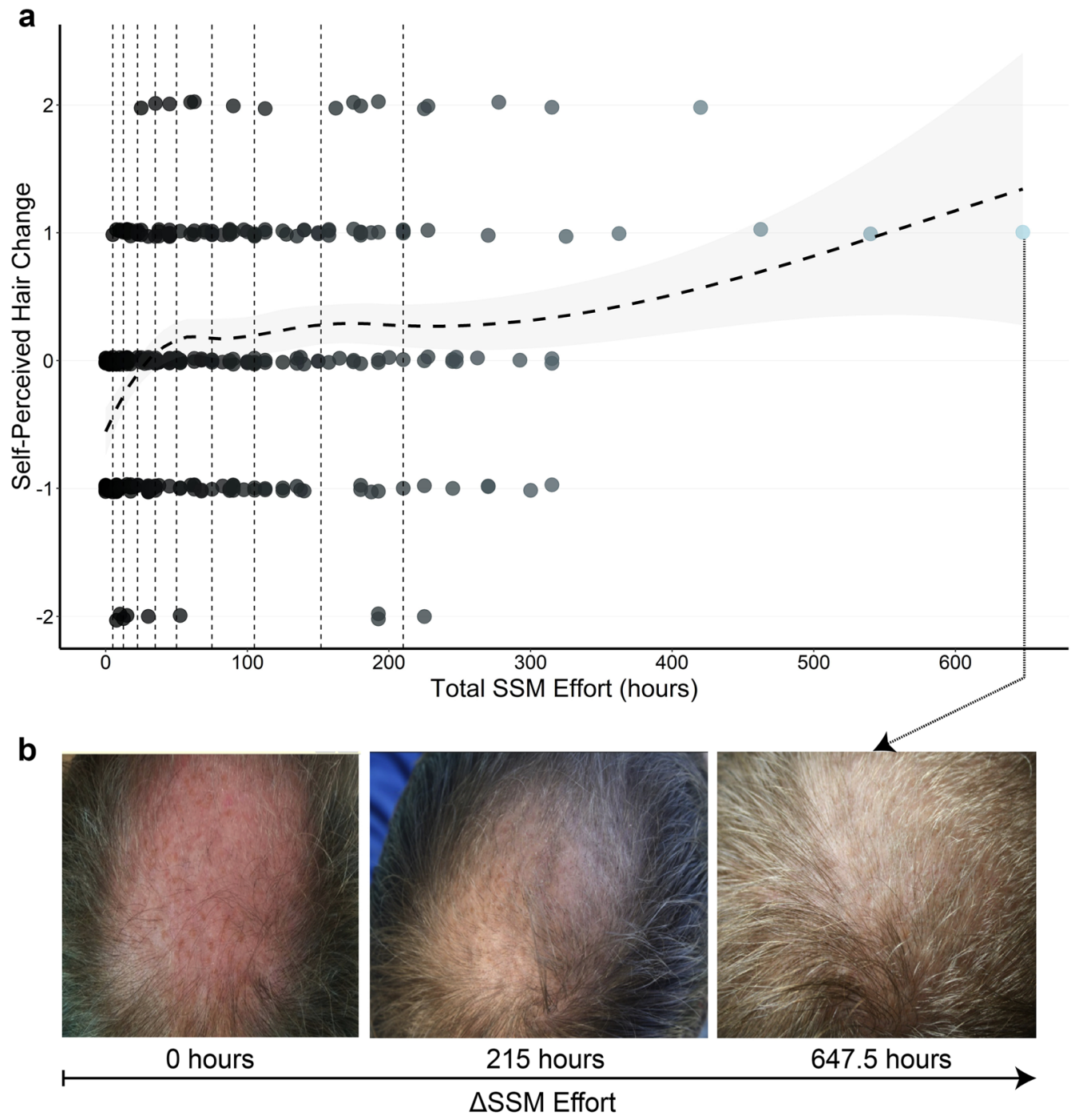

Fig. 2 a Discrete data for reported hair changes for all SSM participants, with vertical dotted lines corresponding to 10-percentile bins of total SSM effort. The dashed trend line corresponds to the average self-perceived hair change

its participants reporting $0-10 \mathrm{~min}$ daily achieved an average of 13.5 months of effort, compared with 2.5 months for those reporting $41+\min$ daily. Accordingly, we considered ways to standardize months of adherence in our analysis. On average, SSM participants reported 7.4 months of effort and hair loss stabilization or greater after 4.5 months (Fig. S5). As such, we selected participants with minimum adherence of $\geq 6$ months, segmented by minutes daily, then evaluated mean hair changes and the for any given SSM effort, with the surrounding grey band indicating an $85 \%$ confidence interval. b Hair change photo assessments of a participant throughout $647.5 \mathrm{~h}$ of SSM effort. Remaining photosets can be found in Fig. S8

percent reporting hair loss stabilization or greater $(0,+1$, or +2$)$. As daily efforts increased from $0-10$ to $41+\min$, hair changes and the percent reporting $\geq 0$ hair changes rose 0.37 points and $15.4 \%$, respectively, but at the expense of a 6.14 -fold increase in average total SSM effort (i.e., an additional $212.8 \mathrm{~h}$ ). This indicates consistent results across analytical approaches, but also that incremental hair changes from higher minutes daily of effort 
came alongside substantially greater time investments.

\section{Effects of SSMs on Self-Perceived Hair Changes for Hair Loss Regions and Gradients}

Due to the small sample size of female SSM participants $(n=29)$, hair loss region and gradient analyses were restricted to males. To determine an association between Norwood gradients and self-perceived hair changes, we grouped male participants by reported gradients of early stage (Norwood 01-02), mid-stage (03-03 vertex), late stage (04-05), and end-stage (06-07). When controlling for total SSM effort, self-perceived hair changes did not vary across stage gradients (Table 1 ).

As AGA gradients progress, hair loss regions overlap. Accordingly, $55.7 \%$ of male SSM participants reported more than one hair loss

Table 1 Parameter estimates for regression models examining predictors of self-perceived hair changes

\begin{tabular}{|c|c|c|c|}
\hline Regression model & $B$ & Standard Error & $P$-value \\
\hline \multicolumn{4}{|l|}{ Massage time } \\
\hline Minutes per day & 0.11 & 0.04 & 0.003 \\
\hline Months & 0.04 & 0.01 & 0.001 \\
\hline Total SSM effort (minutes $\times$ months) & 0.01 & 0.003 & 0.001 \\
\hline \multicolumn{4}{|l|}{ Demographics } \\
\hline Age & 0.04 & 0.04 & 0.30 \\
\hline Gender & -0.04 & 0.18 & 0.81 \\
\hline \multicolumn{4}{|l|}{ Diet } \\
\hline Vegetarian/vegan versus standard American diet & 0.36 & 0.29 & 0.08 \\
\hline Paleo versus standard American diet & 0.07 & 0.15 & 0.66 \\
\hline \multicolumn{4}{|l|}{ Concomitant treatments } \\
\hline Minoxidil & -0.11 & 0.16 & 0.48 \\
\hline Finasteride & -0.02 & 0.27 & 0.95 \\
\hline Microneedling & 0.11 & 0.11 & 0.31 \\
\hline Supplements & 0.05 & 0.10 & 0.62 \\
\hline Topicals & 0.12 & 0.11 & 0.29 \\
\hline \multicolumn{4}{|l|}{ Region and gradient } \\
\hline Diffuse & -0.21 & 0.11 & 0.05 \\
\hline Frontal/temporal & 0.03 & 0.12 & 0.80 \\
\hline Vertex & 0.10 & 0.10 & 0.33 \\
\hline Norwood gradient severity $^{a}$ & -0.09 & 0.06 & 0.17 \\
\hline
\end{tabular}

Total SSM effort was included as a covariate in all models. Concomitant treatments as well as region and gradient variables were coded as $1=$ yes, $0=$ no. Total SSM effort was a covariate in all models assessing demographics, diet, concomitant treatments, and region and gradient variables

${ }^{a}$ Male SSM participants only 


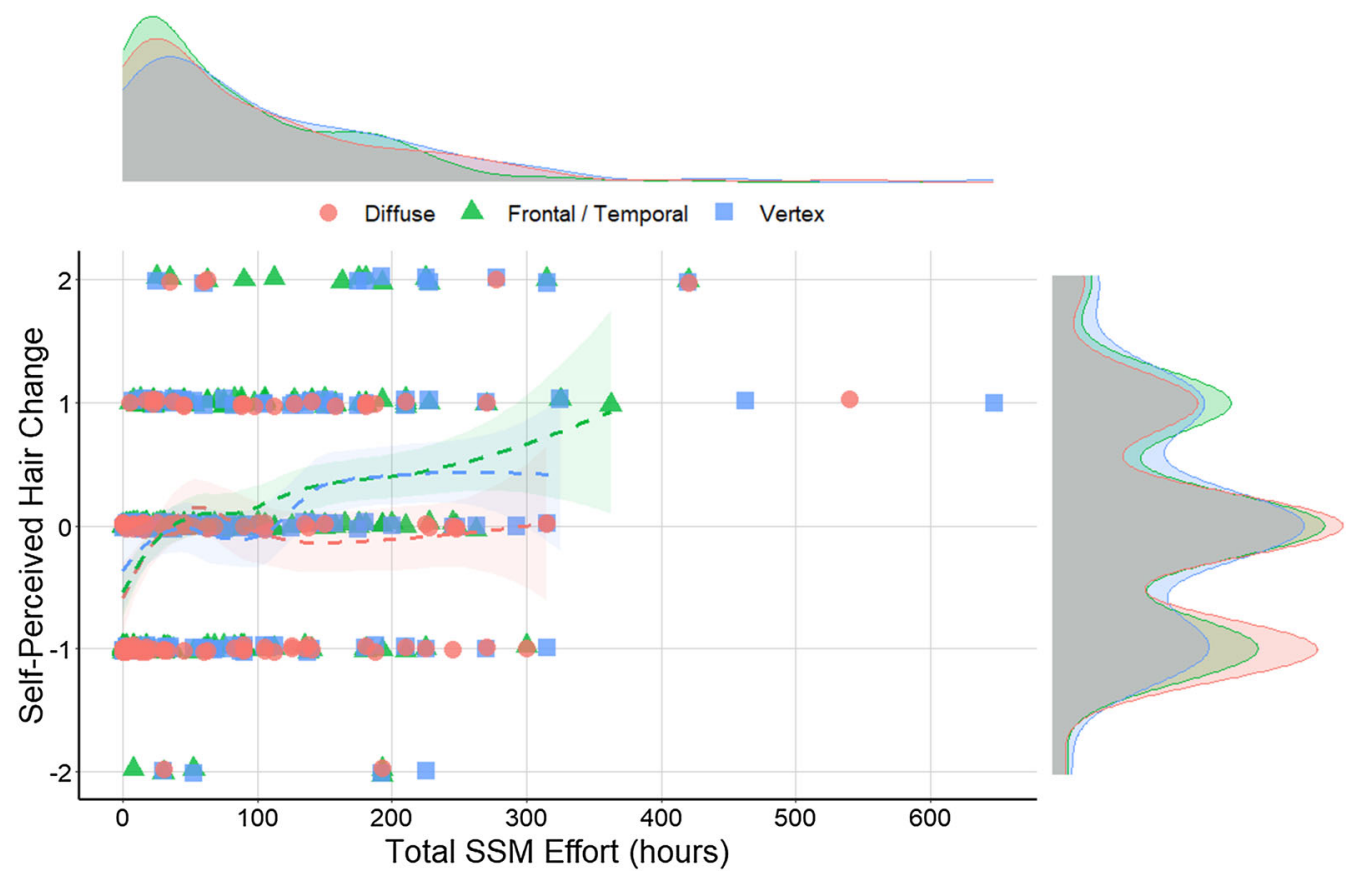

Fig. 3 Discrete data of self-perceived hair changes for SSM participants over total SSM effort, organized by hair loss type. Density distributions show total SSM effort by hair

region. Reported frequencies of frontal/temporal, vertex, and diffuse hair loss were 235 (81.3\%), 134 (46.4\%), and 117 (40.5\%), respectively (Fig. S6). Total SSM effort distributions were relatively consistent across participants regardless of their reported hair loss regions, with mean total SSM efforts of 81.2, 106.2, and $92.2 \mathrm{~h}$ for frontal/temporal, vertex, and diffuse thinning, respectively (Fig. 3). However, selfperceived hair changes varied depending on the regions a participant reported, with lower hair changes for those with diffuse thinning $(-0.11)$ relative to frontal/temporal $(0.03)$ or vertex thinning (0.08). When considering participants in the top $50 \%$ of total SSM effort $(\geq 50 \mathrm{~h}$ ), mean self-perceived hair changes for frontal/ temporal, vertex, and diffuse thinning participants were $0.24,0.19$, and 0.00 , respectively. Notably, for participants in the top $20 \%$ of total SSM effort $(\geq 152 \mathrm{~h})$, this increased to 0.48 , 0.47 , and 0.12 , respectively-indicating positive self-perceived hair changes for participants reporting any hair loss region with continued effort (Fig. 3). loss type (upper chart) and self-perceived hair change count by hair loss type (right of chart)

\section{Effects of SSMs on Self-Perceived Hair Changes for Secondary Parameters}

Finally, we investigated if secondary parameters were associated with self-perceived hair changes in addition to SSM participation: diet; use of minoxidil, finasteride, microneedling, supplements, and topicals; as well as age and gender. All predictors were examined independently while controlling for total SSM effort.

To investigate the association between diet and self-perceived hair change, the standard American diet was used as the reference category against two additional categories to represent plant-based (vegetarian or vegan) and paleo (low-carbohydrate paleo, moderate-carbohydrate paleo, or modified paleo) diets. There was a marginal effect for plant-based diets versus a standard American diet, and no effect for a paleo diet. There were no significant associations between self-perceived hair changes and minoxidil, finasteride, microneedling, supplement, or topical use alongside SSM adherence. 
Neither age nor gender was associated with selfperceived hair change (Table 1).

\section{DISCUSSION}

The results of this survey study show that SSMs improve self-perceived hair changes for SAGASs in a time-dependent manner-with minutes daily, months, and total SSM effort significantly associated with self-perceived hair changes (Table 1). To the best of the authors' knowledge, this is the first study to suggest the therapeutic potential of SSMs for AGA.

Self-perceived hair changes improved with increased minutes daily of massaging, even when controlling for total SSM effort and a minimum number of months of adherence. However, incremental hair changes came at the expense of significantly greater time investment. As such, future SSM participants should consider the tradeoff between response rates, hair changes, and additional hours of SSM dedication. SBTs and mechanotherapies have demonstrated dose-dependent therapeutic response curves-with too little and too much tissue stretch $[13,14]$ or inflammation as either ineffective or counterproductive [19]. A similar relationship may exist for SSMs. Additional research is warranted to determine the optimal minutes daily for which SSMs are most efficient.

Self-perceived hair changes were lower for SSM participants reporting diffuse thinning (Table 1). However, participants with diffuse thinning who reached efforts in the top 50\% $(\geq 50 \mathrm{~h})$ reported a mean self-perceived hair change of 0.00 , with hair changes improving further with continued effort (Fig. 3). This suggests that SSMs may still benefit those with diffuse hair loss, though less so than those with frontal/temporal or vertex thinning.

These results may be explainable through (1) the self-selection of survey participants as AGA sufferers, and (2) the evenly distributed nature of diffuse hair loss. All SSM participants accessed materials targeted toward AGA sufferers. However, not all SSM participants received dermatological diagnoses of AGA. Resultantly, a percentage of diffuse thinners who self-diagnosed as AGA may actually be suffering from conditions which manifest as diffuse thinning-in addition to or in the absence of AGA. Chronic micronutrient deficiencies in ferritin and/or zinc [20], micronutrient excesses of iodine [21], and hypothyroidism can present as diffuse hair loss [22]. Some studies suggest a subclinical hypothyroidism prevalence of up to $10 \%$ in adult populations [23]. Hypothyroidrelated hair thinning differs morphologically from AGA-related hair thinning, and cases of hypothyroid-linked hair loss have been fully reversed following micronutrient supplementation alongside thyroid medication [24]. Consequently, the mechanisms by which SSMs might improve AGA may not apply to non-AGA-related hair loss, which may partly explain the lower assessment scores for diffuse thinners.

Interestingly, a 24-week study on nine nonbalding Japanese men showed that device-mediated SSMs for 4 min daily improved human scalp hair thickness by $10 \%$. However, at the 12-week mark, total hair count temporarily decreased by $5 \%(p<0.05)$, with the decrease disappearing by 24 weeks [14]. The investigators hypothesized that the temporary decrease may have been due to a propensity for SSMs to knock out telogen hairs during massage sessions. Similarly, shedding during SSM participation was commonly reported in open-ended commentary. Due to the evenly distributed nature of diffuse thinning, any temporary increase in hair shedding from SSMs may also result in a more notable decrease in scalp hair coverageparticularly compared to those with frontal/ temporal or vertex hair loss-which may also partly explain the difference in self-perceived hair changes.

While the mechanisms by which SSMs might improve AGA are unknown, overlap may exist between SBTs and soft tissue manipulation therapies. The literature and demonstration video advocated twice-daily SSMs-with an emphasis on evoking erythema (Materials S1, Materials S2). As such, one mechanism might be the repeated activation of wound-healing pathways and subsequent increase in growth factors (i.e., endothelial and VEGF-A), anagen-associated signaling pathways (i.e., Wnt/ $\beta$-catenin), and DP stem cells in balding regions $[9,10]$. 
Interestingly, two-dimensional von Mises models suggest that male AGA patterning overlaps with tensile projections of the galea aponeurotica-the dense fibrous membrane underlying AGA-prone hair follicles-particularly as its connective muscles along the perimeter of the scalp contract. This tension can transmit to scalp hair follicles and may partly explain the pathogenesis of AGA $[25,26]$. Pressure-based hand massages of galea aponeurotica-connected muscles improve tension headaches, likely through release of involuntarily and chronically contracted muscle tissue [27]. Notably, AGA treatments that reduce scalp tension through mechanical offloading (i.e., tension relaxation devices or botulinum toxin injections into galea-linked muscles) improve AGA outcomes [28, 29]. The SSM materials emphasized massaging regions overlying galea aponeurotica-connected muscles, viz. the temporal, parietal, and occipital scalp regions (Materials S1, Materials S2). Accordingly, relaxation of galea-connected muscles may be an additional mechanism by which SSMs improve selfperceived hair changes.

While our findings support previous research on SBTs, SSMs, and human scalp hair, our study should be interpreted in the context of its limitations. A double-blinded, placebo-controlled study is the gold standard for treatment evaluation. As our survey study was retrospective, there was no placebo or control group for age-, gender-, and time-matched non-SSM participants. Evidence suggests that male AGA progresses at a rate of 5\% per year-with periods of acceleration and quiescence-and that without treatment, hair change perceptions should continuously decrease [30]. What remains unknown is the ability of SSM participants to accurately gauge hair changes over shorter time windows (i.e., $<6$ months).

Moreover, self-reported survey data do not allow for objective evaluation of compliance or efficacy. They are also often subject to choicesupportive bias, where participants may be more likely to ascribe positive results (i.e., positive hair changes) to self-selected treatments (i.e., SSMs) [31]. Resultantly, SSM participants retrospectively self-assessing their hair changes may be subject to recall bias for both adherence and outcomes, similar to biases observed in selfreported data for height and weight [32, 33].

Interestingly, our attempts to investigate bias suggested that SSM participants tended to overstate both negative and positive self-perceived hair changes, but that bias washed out across all hair change assessments (Fig. S7). However, these findings are a function of the quality of photographs submitted by participants and are thereby variable (Fig. S8). Dermatological assessments are needed to determine if self-perceptions align with actual hair changes. A clinically controlled setting is more appropriate for future investigations.

Finally, our survey response rate $(19.0 \%)$ is relatively high for an online external survey, but low versus mail-in dermatology-related surveys [34]. While dermatology-related surveys have shown consistent results across survey types (i.e., mail-in, email, or live) and response rates (i.e., 9 to 95\%) [35], it is generally accepted that a higher response rate improves analytical capabilities and that lower response rates may skew results. Given the experimental nature of massage-based therapies for AGA —and the time commitment required for SSM participationwe suspect that many nonparticipants did not take the survey as a result of not attempting the SSMs for an appreciable time period.

\section{CONCLUSIONS}

SSMs improve self-perceived hair changes for SAGASs in a time-dependent manner. Minimum total SSM efforts of $50 \mathrm{~h}$ are recommended to gauge therapeutic potential for participants. Increased minutes daily of massaging improves the odds of self-perceived hair regrowth, but at the expense of significantly greater total SSM effort. Diffuse thinners may perceive marginally lower hair changes relative to those with frontal/temporal or vertex thinning. However, this may be attributable to the higher likelihood of diffuse thinners to have non-AGA-related hair loss, SSM-related telogen hair shedding, and how the even distribution of diffuse thinning may make temporary hair density decreases more noticeable versus frontal/temporal or vertex thinning. Future studies 
should determine if survey findings align with clinical evaluations of AGA subjects, compare SSM efficacy with AGA management drugs, and determine which SSM techniques elucidate the greatest AGA-related hair changes. Finally, the mechanisms and dose-dependent response curves of SSMs deserve further exploration-as either standalone or adjunct AGA treatments.

\section{ACKNOWLEDGEMENTS}

The authors are indebted to all survey respondents for their efforts and participation. The authors would like to thank Michael Molyneux for his encouragement and early guidance in survey design and analyses, and Kaitlyn Fladeboe for her assistance with all data analyses.

Funding. No funding or sponsorship was received for this study or publication of this article. Article processing charges were funded by the authors.

Authorship. All named authors meet the International Committee of Medical Journal Editors (ICMJE) criteria for authorship for this article, take responsibility for the integrity of the work as a whole, and have given their approval for this version to be published.

Author Contributions. RSE Jr. designed the study, supervised the research, and wrote the paper. JMB analyzed the data, created the figures, and assisted in writing the paper.

Disclosures. RSE Jr. operates a commercial website which intends to provide education about and services for some of the concepts in this paper. JMB has nothing to disclose.

Compliance with Ethics Guidelines. All procedures performed in studies involving human participants were in accordance with IntegReview IRB, who granted an exemption from Institutional Review Board oversight in accordance with 45 CFR 46.101(b) and with the 1964 Helsinki declaration and its later amendments or comparable ethical standards.
Informed consent was obtained from all individual participants included in the study.

Data Availability. The datasets analyzed during the current study are available in the supplementary material.

Open Access. This article is distributed under the terms of the Creative Commons Attribution-NonCommercial 4.0 International License (http://creativecommons.org/licenses/ by-nc/4.0/), which permits any noncommercial use, distribution, and reproduction in any medium, provided you give appropriate credit to the original author(s) and the source, provide a link to the Creative Commons license, and indicate if changes were made.

\section{REFERENCES}

1. Gan DC, Sinclair RD. Prevalence of male and female pattern hair loss in Maryborough. J Investig Dermatol Symp Proc. 2005;10(3):184-9.

2. Cash TF. The psychosocial consequences of androgenetic alopecia: a review of the research literature. Br J Dermatol. 1999;141(3):398-405.

3. Lolli $\mathrm{F}$, et al. Androgenetic alopecia: a review. Endocrine. 2017;57(1):9-17.

4. Yoo HG, et al. Perifollicular fibrosis: pathogenetic role in androgenetic alopecia. Biol Pharm Bull. 2006;29(6):1246-50.

5. Kaufman KD, et al. Long-term treatment with finasteride $1 \mathrm{mg}$ decreases the likelihood of developing further visible hair loss in men with androgenetic alopecia (male pattern hair loss). Eur J Dermatol. 2008;18(4):400-6.

6. Kaufman KD, Finasteride Male Pattern Hair Loss Study Group, et al. Finasteride in the treatment of men with androgenetic alopecia. J Am Acad Dermatol. 1998;39(4):578-89.

7. Olsen EA, et al. A multicenter, randomized, placebo-controlled, double-blind clinical trial of a novel formulation of $5 \%$ minoxidil topical foam versus placebo in the treatment of androgenetic alopecia in men. J Am Acad Dermatol. 2007;57(5):767-74.

8. Mapar MA, Omidian M. Is topical minoxidil solution effective on androgenetic alopecia in routine 
daily practice? J Dermatolog Treat. 2007;18(5):268-70.

9. Khatu SS, et al. Platelet-rich plasma in androgenic alopecia: myth or an effective tool. J Cutan Aesthet Surg. 2014;7(2):107-10.

10. Fertig RM, et al. Microneedling for the treatment of hair loss? J Eur Acad Dermatol Venereol. 2018;32(4):564-9.

11. Bharti J, et al. Scalp threading with polydioxanone monofilament threads: a novel, effective and safe modality for hair restoration. J Eur Acad Dermatol Venereol. 2017;31(11):e492-3.

12. Enshell-Seijffers D, et al. $\beta$-Catenin activity in the dermal papilla regulates morphogenesis and regeneration of hair. Dev Cell. 2010;18(4):633-42.

13. Best TM, Gharaibeh B, Huard J. Stem cells, angiogenesis and muscle healing: a potential role in massage therapies? Postgrad Med J. 2013;89(1057):666-70.

14. Koyama T, et al. Standardized scalp massage results in increased hair thickness by inducing stretching forces to dermal papilla cells in the subcutaneous tissue. Eplasty. 2016;16:e8.

15. Andrzejewski W, et al. Effects of synergistic massage and physical exercise on the expression of angiogenic markers in rat tendons. Biomed Res Int. 2014;2014:878095.

16. Patel KG, Kobayashi M, Best T, Huard J. No. 63 immediate massage stimulates muscle regeneration angiogenesis and decreases fibrosis after muscle injury (Encore Presentation). PM\&R 2014;6(8):S95. https://doi.org/10.1016/j.pmrj.2014.08.311.

17. Andrzejewski W, et al. Massage may initiate tendon structural changes-a preliminary study. Vivo. $2015 ; 29(3): 365-9$.

18. Cho YS, et al. The effect of burn rehabilitation massage therapy on hypertrophic scar after burn: a randomized controlled trial. Burns. 2014;40(8):1513-20.

19. Agarwal S, et al. A central role for the nuclear factorkappaB pathway in anti-inflammatory and proinflammatory actions of mechanical strain. FASEB J. 2003;17(8):899-901.

20. Rushton DH. Nutritional factors and hair loss. Clin Exp Dermatol. 2002;27(5):396-404.

21. Chapman RS, Main RA. Diffuse thinning of hair in iodide-induced hypothyroidism. Br J Dermatol. 1967;79(2):103-5.
22. Keen MA, Hassan I, Bhat MH. A clinical study of the cutaneous manifestations of hypothyroidism in Kashmir valley. Indian J Dermatol. 2013;58(4):326.

23. Schubel J, et al. Latent hypothyroidism in adults. Dtsch Arztebl Int. 2017;114(25):430-8.

24. Betsy A, Binitha M, Sarita S. Zinc deficiency associated with hypothyroidism: an overlooked cause of severe alopecia. Int J Trichol. 2013;5(1):40-2.

25. Tellez-Segura R. Involvement of mechanical stress in androgenetic alopecia. Int $\mathrm{J}$ Trichology. 2015;7(3):95-9.

26. English RS Jr. A hypothetical pathogenesis model for androgenic alopecia: clarifying the dihydrotestosterone paradox and rate-limiting recovery factors. Med Hypotheses. 2018;111:73-81.

27. Smith LS. Evaluation and management of the muscle contraction headache. Nurse Pract. 1988;13(1):20-3.

28. Toshitani S, et al. A new apparatus for hair regrowth in male-pattern baldness. J Dermatol. 1990;17(4):240-6.

29. Freund BJ, Schwartz M. Treatment of male pattern baldness with botulinum toxin: a pilot study. Plast Reconstr Surg. 2010;126(5):246e-8e.

30. Sinclair R. Male pattern androgenetic alopecia. BMJ. 1998;317(7162):865-9.

31. Mather M, Johnson MK. Choice-supportive source monitoring: do our decisions seem better to us as we age? Psychol Aging. 2000;15(4):596-606.

32. Schoeller DA. Limitations in the assessment of dietary energy intake by self-report. Metabolism. 1995;44(2 Suppl 2):18-22.

33. Spencer EA, et al. Validity of self-reported height and weight in 4808 EPIC-Oxford participants. Public Health Nutr. 2002;5(4):561-5.

34. Filip JC, et al. Mail surveys can achieve high response rates in a dermatology patient population. J Invest Dermatol. 2004;122(1):39-43.

35. Glazer A, et al. Comparison of survey modality and response rate in dermatologists' perceptions and opinions of sunscreens. SKIN J Cutan Med. 2018;2(2):5. 